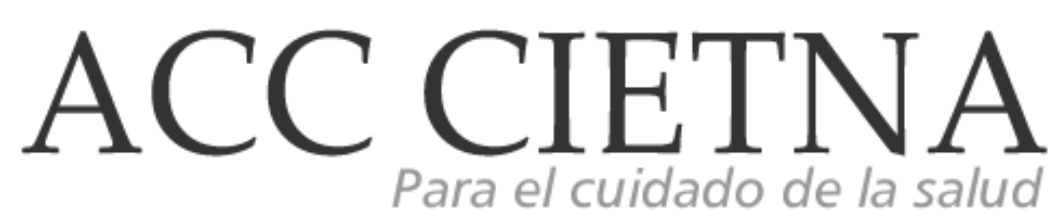

https://doi.org/10.35383/cietna.v8i1 1359

\title{
Experiencias en el uso del internet en estudiantes de enfermería de una universidad privada
}

\author{
Cabrejos Solis Sandra Nevenka', Guerrero Quiroz Elizabeth Soledad², Diaz Manchay Rosa Jeuna
}

INFORMACIÓN DEL ARTÍCULO RESUMEN

Historia del artículo:

Recibido el 17 de septiembre de 2020

Aceptado el 11 de febrero de 2021

Palabras clave:

Experiencia (D008016)

Internet (D020407)

Estudiantes de enfermería (D013338)
Objetivo: Describir las experiencias en el uso de internet de los estudiantes de Enfermería de una universidad privada de Chiclayo, 2019. Método: Esta investigación fue cualitativa descriptiva exploratoria. El tamaño de la muestra fue de 12 estudiantes del primer año que estudian en la escuela de enfermería de la Universidad Católica Santo Toribio de Mogrovejo, obtenidos por la técnica de saturación y redundancia, previo consentimiento informado. Para la obtención de datos se aplicó la entrevista semiestructurada, elaborada por la investigadora y validada por juicio de expertos. Los datos se procesaron con análisis de contenido, se tuvo en cuenta los principios éticos y de rigor científico. Resultados principales: Dos categorías: a) Uso de internet como soporte educativo: campus virtual, búsqueda de información y trabajos grupales y b) Uso de internet como medio de comunicación y entretenimiento: riesgos y repercusiones. Conclusión general: Los estudiantes de enfermería utilizan al internet como una herramienta necesaria y muy útil para el contexto universitario, sin embargo, también lo usan para mantenerse comunicados con su familia y amigos, y para el ocio, pero han experimentado repercusiones en su salud (trastornos del patrón del sueño como el insomnio, los ojos rojos y cansados, cansancio físico), y en lo social (ciberbullying).

1Bachiller de la Escuela de Enfermería de la Universidad Católica Santo Toribio de Mogrovejo, Chiclayo, Perú. Email: sandra.cabrejoss@gmail.com. ORCID: https://orcid.org/0000-0002-9773-0303

2Doctora en Ciencias de Enfermería. Docente de la Escuela de Enfermería de la Universidad Católica Santo Toribio de Mogrovejo, Chiclayo, Perú. Email sguerrero@usat.edu.pe. ORCID: https://orcid.org/0000-0002-8094-5711

${ }^{3}$ Doctora en Ciencias de Enfermería. Docente de la escuela de Enfermería de la Universidad Católica Santo Toribio de Mogrovejo, Chiclayo, Perú. Email: rdiaz@usat.edu.pe, ORCID: https://orcid.org/0000-0002-2333-7963 
Experiences in the use of the Internet in nursing students from a private university

ABSTRACT

Keywords:

Experience (D008016)

Internet (D020407)

Nursing students (D013338)
Objective: To describe and understand the experiences in the use of the Internet of Nursing students from a private university in Chiclayo, 2019. Method: This research was qualitative descriptive exploratory. The sample size was 12 first-year students studying at the nursing school of the Santo Toribio de Mogrovejo Catholic University, obtained by the saturation and redundancy technique, with prior informed consent. To obtain data, the semi-structured interview was applied, prepared by the researcher and validated by expert judgment. The data was processed with content analysis, ethical principles and scientific rigor were taken into account. Main results: Two categories: a) Use of the Internet as an educational support: virtual campus, information search and group work and b) Use of the Internet as a means of communication and entertainment: risks and repercussions. General conclusion: Nursing students use the internet as a necessary and very useful tool for the university context, however, they also use it to keep in touch with their family and friends, and for leisure, but they have experienced repercussions on their health (pattern disorders sleep such as insomnia, red and tired eyes, physical fatigue), and socially (cyberbullying).

\section{Introducción}

En todo el mundo, más de 4.5 mil millones de personas ahora usan Internet, siendo China en donde más lo usan, de este modo el internet se convierte en una parte indispensable de la vida cotidiana de las personas porque facilita la comunicación, el estudio y el entretenimiento'. En México, su población llega a un $71 \%$ de alcance, y, el $67 \%$ está conectado a internet todo el día². En Perú durante el tercer trimestre del año 2019, el $46.7 \%$ ingresó a internet desde su celular, el $79.4 \%$ lo usa diariamente, y los que más usan internet tiene entre 19 a 24 años ${ }^{3}$.

En España el estudio de Elche ${ }^{4}$ establece que los estudiantes universitarios usan el internet para situaciones académicas, comunicativas y por distracción. En Ecuador, Mininguano ${ }^{5}$, muestra que los estudiantes universitarios de su estudio emplean todos están registrados en al menos una red social y participan en ellas más de una hora por día. Mientras que en el contexto colombiano el porcentaje de universitarios que usan internet de cinco a más horas durante el día es de $49 \%$.

En Perú, Valer ${ }^{7}$ concluyó que para las actividades académicas los estudiantes usaron el internet en diferentes niveles, donde el $52.9 \%$ fue de nivel alto y el $47.1 \%$ de nivel intermedio, pero un mal manejo de ello trae consigo riesgos. Debido al incremento masivo del internet, su uso inadecuado por los estudiantes puede conllevar al usuario a problemas de adicción o sentimentales y bajo rendimiento académico $^{8}$. El $95.3 \%$ de los universitarios que usan internet tienen problemas de salud como el mal dormir, depresión, mala memoria, y relaciones familiares deficientes ${ }^{9}$.

Por lo expuesto se tuvo como objetivo: Describir las experiencias en el uso de internet de los estudiantes de Enfermería de una universidad privada de Chiclayo, 2019. 


\section{Metodología}

Se llevó a cabo una investigación cualitativa descriptiva exploratoria 10 a los estudiantes universitarios pertenecientes del primer año de la escuela de enfermería de la universidad Católica Santo Toribio de Mogrovejo (USAT). La población fue de 93 estudiantes. El tamaño de la muestra fue de 12 estudiantes de enfermería, fue determinada por la técnica de saturación y redundancia de datos; y el muestreo fue no probabilístico. Fueron solteros, sus edades oscilaban entre 17-20 años. No se incluyeron a estudiantes que sean técnicos de enfermería, o que sean madres de familia o quienes estén adelantando cursos. Fueron entrevistados en setiembre del 2019, previa firma del consentimiento informado.

Los datos se recolectaron a través de una guía de entrevista semiestructurada que estuvo constituida de 7 preguntas, fue sometida a una revisión de tres expertos. Se hizo la prueba piloto con dos estudiantes de otra universidad privada. Luego se obtuvo la aprobación del Comité de Ética en Investigación de la Facultad de Medicina de la Universidad Católica Santo Toribio de Mogrovejo (Resolución $\mathrm{N}^{\circ} 157295$ ) y de la autorización de la Dirección de la escuela de enfermería-USAT, se contactó con los estudiantes y según la disponibilidad se acordaba la fecha y hora para ejecutar las entrevistas. Se les explicó a los participantes sobre los objetivos de la investigación, y luego se su consentimiento informado se procedió a grabarlas, duro aproximadamente 15 minutos.

Para el análisis de datos se realizó el preanálisis, donde se realizaron las escuchas de los testimonios de los estudiantes de enfermería muy meticulosamente para ser parte de y lograr estar inmerso para hacer un buen análisis. Posterior a ello la codificación, aquí se convirtió del lenguaje EMIC al ETIC logrando obtener de los discursos más significativos, identificando una frase o idea principal de cada respuesta para develar el objeto d estudio. Por último, en la tercera etapa se categorizó, haciendo un ordenamiento y clasificación por semejanzas obteniendo dos categorías.

Se aplicaron los principios de Elio Sgreccia ${ }^{11}$, el principio de libertad y responsabilidad: los estudiantes de enfermería fueron libres y responsables de su decisión en participar, lo cual fue manifestado a través del consentimiento informado. Se los identificó con códigos: $\operatorname{EENF(1)~}$ hasta EENF(12) para proteger su identidad. Y la subsidiariedad se aplicó en la presente investigación, puesto que en el futuro este estudio podrá ser utilizado como referencia para otras investigaciones con la misma temática.

Una de las limitaciones metodológicas del estudio es que solo ha consignado el punto de vista de los estudiantes, por ello se sugiere para posteriores estudios se incluya a los docentes.

\section{Resultados, análisis y discusión}

\section{Categoría 1: Uso de internet como soporte educativo: campus virtual, búsqueda de información y trabajos grupales}

En el contexto universitario, la relación internetpersona se da de manera natural, siendo parte de la vida de estos jóvenes ${ }^{12}$. Actualmente el uso del internet ha impactado más a los universitarios, pues casi todos se conectan a internet, y es muy necesario para su desempeño académico ${ }^{13}$.

Para los universitarios del estudio, es indispensable utilizar el internet para acceder a su campus virtual, buscar información o reunirse en línea para realizar los trabajos académicos. En sí, acceden a las plataformas virtuales $y$, revisan el material que cuelga el docente $y$ ver sus calificaciones, como se evidencia en los siguientes testimonios:

\footnotetext{
"Me conecto para hacer uso del campus virtual porque las profesoras cuelgan las guías de trabajo que debemos desarrollarlas para las clases" EENF (2)
} 
"veo mis calificaciones y como va mi avance académico, en el campus virtual de la universidad, y que tareas están pendientes para colgar...reviso las presentaciones de PPT y la información que cuelgan las docentes en el aula virtual' EENF (9)

Los estudiantes de enfermería hacen uso del campus virtual para desarrollar guías y trabajos que las profesoras cuelgan de las clases correspondientes, además les permite tener información acerca de su rendimiento académico, con la revisión de sus notas, consideran que este medio va de la mano con la parte formativa y la actualización de sus conocimientos científicos para su desempeño profesional. Resultados similares al estudio de Martínez, Pérez, Martínez ${ }^{14}$ donde el internet favorece el uso del entorno virtual, esto facilita el manejo de la información y permite que cada estudiante conozca el avance de sus calificaciones y el docente pueda hacer seguimiento y retroalimentación. Chalela, Valencia, Bermúdez y Ortega ${ }^{15}$ concluye que los estudiantes gracias al internet ingresan y usan sus plataformas virtuales, además perciben que es fácil el manejo de la información impartida virtualmente.

Por otro lado, los estudiantes refieren que el internet proporciona información actualizada, rápida y accesible, lo cual es muy beneficioso para ellos puesto que les dejan trabajos y de esta manera pueden presentar y entregar a tiempo sus tareas, como lo detallan en los siguientes testimonios:

"puedo realizar mis trabajos de la universidad mediante la búsqueda fácil por internet o por la biblioteca de la universidad" EENF (2), "con el internet tengo acceso a libros digitales" EENF(l 1)

"para hacer trabajos, investigar, para ver videos de cirugías, de manera que en mis ratos libres me entretengo aparte de estudiar." EENF (4) "...ahorro al ya no ir a la biblioteca, el internet está a la mano y accesible, saco artículos para mis tareas" EENF(8)

“... El internet resulta mucho más rápida que antes, ya no vamos con frecuencia a las bibliotecas por lo que encontramos libros virtuales, más accesibles que los empastados" EENF(6)

Los estudiantes dan a conocer que el internet les permite una búsqueda efectiva con tan solo dar un clic, ya no es necesario que pasen horas de horas en la biblioteca porque en el internet encuentran libros actualizados, artículos, etc., asimismo, en la página web de la universidad, tienen acceso a base de datos, revistas, tesis y libros virtuales las diferentes bases de datos en internet permite encontrar revistas, libros actualizados, artículos, etc. relacionados a su búsqueda de manera gratuita, desde la comodidad de su casa o desde cualquier otro lugar. Resultados similares al estudio de Linne ${ }^{16}$, indica que los estudiantes bajan información para sus trabajos, ven la programación de sus exámenes y todo ello lo hacen desde sus viviendas ya que cuentan con sus laptops y/o computadoras. Para, los estudiantes el internet lo consideran es útil para realizar sus trabajos dejados en clases; además pueden hallar libros incluso gratuitamente, trabajos para hacer investigación ${ }^{17}$.

Al mismo tiempo el internet ha facilitado la creación de trabajos de equipo en línea, lo cual es de gran beneficio porque se puede trabajar con más personas, aprendiendo a intercambiar roles y a mejorar la comunicación, como lo demuestran los siguientes te

"...lo utilizo para conversar con mis amigos o grupos de estudios de la universidad para poder hacer los trabajos grupales..." EENF(1)

“...me permite realizar mis trabajos grupales contactándome con mis compañeros de estudios a través del 
WhatsApp, proyectar los trabajos de la universidad, pdf virtuales, power point virtuales también..." EENF(l l)

En estos discursos los alumnos manifiestan que les permite hacer sus trabajos grupales en línea, usan WhatsApp. Según Neves et al ${ }^{18}$, el internet permite múltiples beneficios como el crear trabajos de equipos en línea, ganar capacidad para su organización propia, logrando así la mejoría de su desarrollo como estudiante universitario. Barbosa, Barbosa y Villamizar ${ }^{19}$ establece que los trabajos en equipo se han virtualizado. El estudio de Lynch y Calsín 20 encontró que a través del internet los universitarios usan WhatsApp para los trabajos en equipo.

Categoría 2: Uso de internet como medio de comunicación y entretenimiento: riesgos y repercusiones

El internet es un recurso para estar en comunicación ocupando la mayoría del tiempo de las personas, creando nuevos lazos entre ellos de manera virtual ${ }^{21}$. La influencia de este medio de comunicación se ha producido con mayor nivel en la población universitaria que han encontrado un medio perfecto de comunicación, relación, diversión y satisfacción de la curiosidad 22. Como lo manifiestan en los siguientes testimonios:

"...como entretenimiento también me conecto a través de las redes sociales" EENF(2) "lo uso para conversar con mamá, quien está un poco lejos y de esa manera nos mantenemos comunicadas, también con mis hermanitos pequeños" $\operatorname{EENF(3)}$

“...para chatear, para entretenerme a través de las redes sociales Facebook, WhatsApp, juego y como Netflix se ve desde el celular veo mis series hasta que se terminen mis megas" EENF(5)

En este estudio los universitarios también usan el internet para entretenerse, comunicarse con amistades y familiares sobre todo cuando viven en diferentes ciudades. Se recalca que estos estudiantes fueron del primer año, quienes por sus edades están más tiempo en internet. Esto se corrobora con el estudio de Segura, Neciosup, Díaz, Alcántara y Díaz ${ }^{23}$ encontraron que, en la población de 18 a 24 años de edad, utiliza el internet para comunicarse en un $97.4 \%$ y para lo lúdico un $84,2 \%$. Así mismo, la mayor proporción de internautas corresponde al $95 \%$ de los jóvenes de 16 a 34 años, los estudiantes y aquellas personas con estudios superiores se conectan diariamente a internet ${ }^{24}$.

Por otro lado, así como el internet da múltiples beneficios, este puede traer innumerables problemas por su mal manejo. De tal manera que, el internet también ha generado dependencias, puesto que lo que antes les parecía atractivo ahora ya no lo es, por estar tan inmersos en el mundo virtual, dejando de lado su verdadera vida, generando problemas con su propia familia, problemas sociales y emocionales ${ }^{25}$. Develado en los siguientes discursos:

“...he estado varios días con insomnio, fue producto del uso indiscriminado y no me daba cuenta hasta que me llevaron al médico, además de mis ojos rojos $y$ cansados..." EENF(4)

“...he llegado a jugar en las madrugadas malográndome mi propio sueño llegando a alterarlo y sufrir de insomnio..." EENF(6)

Los estudiantes de enfermería como lo podemos evidenciar tienen una mala calidad del sueño llegando así al insomnio, uno de los principales trastornos del sueño. Según el estudio de Domínguez, Gonzales y Ramírez ${ }^{26}$ los estudiantes reconocen lo que puede causar la demanda alta de uso de internet, como problemas de visión, problemas en la columna vertebral y el insomnio, ojos rojos. Lo que causa el insomnio primordialmente es que disminuye la memoria por ejemplo cuando la atención es compleja ${ }^{27}$. 
Como estudiantes de enfermería, deben saber de las consecuencias que el insomnio le traerá a largo plazo y que podría afectar su rendimiento. Pero, no se dan cuenta del riesgo, de la repercusión que puede traer el uso del internet de manera deliberada como por ejemplo problemas en la salud, psicosocial, etc. Ellos manifiestan que pierden tiempo jugando y hasta tal vez tengan adicción al internet, tal como se testifica a continuación:

“...Me conecto todos los días y todo el día a internet...siempre juego en línea me paso horas en ello distrayéndome de mis estudios he llegado a jugar en las madrugadas malográndome mi propio sueño llegando a alterarlo y sufrir de insomnio..." EENF(6)

\section{“...Me conecto tres o cuatro veces al día} o más, mi celular tiene internet y me permite estar conectado casi todo el día..."EENF(3)

Pero por otro lado se encuentra el ciberdelito o ciberdelincuencia pues están más expuestas las informaciones de las personas 28 . En cuanto al ciberbullying los autores Rodríguez y Rivadulla 29 indican según sus resultados que los jóvenes sufren acoso a través de Internet (108 mujeres y 72 hombres), la duración del acoso sufrido suele ser de una semana o menos y la forma en la que les acosan a través de Internet suele ser por chats. Como se evidencian los siguientes testimonios:

“...Me han hecho bullying, estaba deprimida, mi autoestima baja a consecuencia de ello tuve que ir a terapia psicológica. Otro aspecto negativo es que a través de las páginas de Facebook o Instagram me han hablado personas desconocidas para invitarme a salir llegando a hostigarme... "EENF(2)

“... Otra experiencia negativa fue que hace unos meses a través de Facebook me acosaron y fue la pareja de mi ex enamorado para hacerme quedar mal ante mucha gente y lo hizo porque todos esos comentarios salían como anónimos no se podía ver que era ella..." EENF(l l)

Estos jóvenes no miden el tiempo usado en internet y mucho menos los peligros a los que están expuestos a través de sus redes sociales cuando publican fotos en bikinis, o fotos enseñando la fachada de sus casas y no miden el peligro al que se exponen, porque hay gente que se aprovecha de esto para publicarlo en páginas pornográficas o pueden ser víctimas de robos. Otra de las consecuencias de invertir tanto tiempo en internet es el ciberbullying ya que este acoso es más fácil que sea a través del internet ya que los acosadores están detrás de una pantalla.

\section{Conclusiones}

El internet es de gran contribución para la formación universitaria porque brinda información de fácil acceso y de manera rápida, permite hacer reunirse en línea para hacer trabajos. Asimismo, usan el internet para interactuar con su campus virtual, subir trabajos, ver el material que cuelga el docente y revisar sus calificaciones.

El internet es útil en el aspecto comunicativo, pues permite la conexión rápida con su círculo social y familiar, a través de las diferentes aplicaciones que ellos mencionan como: WhatsApp, Facebook, etc. Existen varias opciones para el ocio como ver películas en Netflix, descargar música, y jugos en línea, etc. Estas redes sociales están ligadas al uso comunicativo y al entretenimiento, pero el uso indiscriminado del internet llega a afectar la salud física (trastornos del sueño como el insomnio, ojos rojos, y cansancio físico), y en el aspecto social (ciberbullying). Los estudiantes no se dan cuenta de las consecuencias tanto en el presente como en el futuro, de tal manera que, si como estudiante de ciencias de la salud ahora ya les genera dichas afecciones, si eso no se frena, cuando ya sean trabajadores de salud podrían cometer errores a la hora de trabajar por no estar concentrados en su labor, sino navegando por internet. 


\section{Bibliografía}

1. Fernández J. Digital 2020. We are social [Internet]. 2020 [consultado 20 Abr 2021]. Disponible

en:

https://wearesocial.com/digital-2020

2. Asociación de Internet MX [Internet]. México: Asociación de Internet MX; 2019 [consultado Agos 2019]. $15^{\circ}$ Estudio sobre los Hábitos de los Usuarios de Internet en México 2018; 1-25 pág. Disponible en: https://irpcdn.multiscreensite.com/81280eda/files/uplo aded/1 1 \%2BEstudio\%2Bsobre\%2Blos\%2BHa_bit os\%2Bde\%2Blos\%2BUsuarios\%2Bde\%2BInternet\% 2Ben\%2BMe_xico\%2B2019\%2Bversio_n\%2Bpu_b lica.pdf

3. Ruiz R. Estadísticas de las Tecnologías de la Información y Comunicación en los Hogares [Internet]. Lima: INEI; 2019 [consultado Agos 2019]. Disponible en: https://www.inei.gob.pe/media/MenuRecursiv o/boletines/03-informe-tecnicon03_tecnologias-de-informacion-abr-mayjun01 9.pdf

4. Elche M, Yubero S. Empleo de internet en los estudiantes de la universidad de castilla-la mancha. ResearchGate. 2017 [consultado 20 Abr 2020]; 1-9. Disponible en: https://www.researchgate.net/publication/328 383346_Empleo_de_Internet_en_los_estudiante s_de_la_Universidad_de_Castilla-La_Mancha

5. Mininguano D. Uso del internet, las redes sociales y su impacto en el rendimiento académico de los estudiantes de la carrera de comunicación social de la universidad técnica de Ambato. [Tesis de pregrado en Internet]. Ambato: Universidad Técnica de Ambato; 2017. [consultado $25 \mathrm{Abr} 2019$ ]. 146 p. Disponible en: http://repo.uta.edu.ec/bitstream/123456789/ 25343/1/FJCS-CS-452.pdf

6. González G. Uso problemático de internet y calidad de vida en universitarios de una institución privada confesional de Medellín, Colombia, 2017. Revista de Investigación Universitaria [Internet]. 2018 [consultado 20
Abr 2019]; 6(2): 62- 85. Disponible en: https://doi.org/10.17162/riu.v6i2.1075

7. Valer M. Uso del internet en estudiantes del nivel secundario de la IEP "Jesús divino maestro n 20194 del distrito de Santa Cruz de Flores2017. [Tesis de pregrado en Internet]. Lima: Universidad César Vallejo; 2019 [consultado 25 abril 2019]. 58 p. Disponible en: https://hdl.handle.net/20.500.12692/28541

8. Rengifo C, Mamani L, Vilca W. Funcionamiento familiar y adicción a internet en estudiantes de una institución educativa pública de Lima Norte. Revista Científica de Ciencias de la Salud [internet]. 2016 [consultado 22 abril 2019]; 8(2):9-17. Disponible en: https://revistas.upeu.edu.pe/index.php/rc_sal ud/article/view/245/245

9. Cañon S, Castaño J, Hoyos D, Jaramillo J, Leal D et all. Uso del internet y su relación con la salud en estudiantes universitarios de la ciudad de Manizales. Caldas - Colombia. Archivos de Medicina (Col) [internet]. 2016 [consultado 24 abril 2019]; 16(2): 312-325. Disponible en: https://www.redalyc.org/pdf/2738/27384994 5010.pdf

10.Grove S, Gray J, Burns N. Investigación en enfermería: Desarrollo de la práctica enfermera basada en la evidencia. 6ta ed. Barcelona: EdiDe; 2016. 539 p.

11.Sgreccia E. Manual de bioética. Madrid: Biblioteca de Autores Cristianos; 2009

12.Yus F. Los efectos de Internet y las redes sociales en el español. Boletín de la Academia Puertorriqueña de la Lengua Española [Internet]. 2017 [consultado 10 Nov 2019]; 55-76. Disponible en: http://hdl.handle.net/10045/76327

13.Torres J, Duart J, Gómez H, Marín I, Segarra V. Usos de Internet y éxito académico en estudiantes universitarios. Comunicar [Internet]. 2016 [consultado 15 Nov 2019]; 14(48): $\quad 61-70 . \quad$ Disponible en: https://dialnet.unirioja.es/servlet/articulo?codi go $=5563116$

14. Martínez P, Pérez J, Martínez M. Las TICs y el entorno virtual para la tutoría universitaria. 
Educación XX1 [Internet]. 2016 [consultado 15 Nov 2019]; 19 (1): 287-310. Disponible en: http://e-

spacio.uned.es/fez/view/bibliuned:EducacionX XI-2016-19-1-5060

15.Chalela S, Valencia A, Bermúdez J, Ortega C. Percepciones estudiantiles acerca del uso de nuevas tecnologías en instituciones de Educación Superior en Medellín. Revista Lasallista de Investigación [Internet]. 2016 [consultado 17 Nov 2019]; 13(2): 151-162. Disponible en: http: / / www.scielo.org.co/scielo.php?pid=S179 4-

$44492016000200015 \&$ script $=$ sci_abstract $\&$ tln $\mathrm{g}=\mathrm{pt}$

16.Linne J. Estudiar en Internet 2.0. Prácticas de jóvenes universitarios de la Ciudad de Buenos Aires. Comunicación y Sociedad [Internet]. 2015 [consultado 15 Nov 2019]; (23): 195-213. Disponible en: http://www.scielo.org.mx/pdf/comso/n23/n2 3a9.pdf

17.Santillán J, Guerra J. Incidencia del tiempo de uso de internet en el rendimiento académico en un grupo de estudiantes universitarios de la ciudad de Riobamba (Ecuador). Revista Científica y Tecnológica UPSE [Internet]. 2015 [consultado 16 Nov 2019]; 2(3): 7. Disponible en: https://doi.org/10.26423/rctu.v2i3.51

18. Neves S, Pinto P, Gomes A, Santos J, Viseu J, Rosa J, et al. Proceedings of the II International Congress on Interdisciplinarity in Social and Human Sciences [Internet]. Faro: CIEO; 2017 [consultado 27 Dic 2019]. Capítulo 1. Utilización de internet en la promoción de estrategias de éxito en la universidad. Disponible en: http://hdl.handle.net/10400.1/10086

19.Barbosa J, Barbosa J, Villamizar J. Trabajo colectivo y sistematización de experiencias educativas: aportes desde la formación en modalidad virtual (Universidad Industrial de Santander, Colombia). Ciencia, cultura y sociedad [Internet]. 2016 [consultado $23 \mathrm{Abr}$
2020]; 3(2): 55-63. Disponible en: https://doi.org/10.5377/ccs.v3i2.5988

20.Lynch A, Calsín J. Uso académico de WhatsApp en grupos de estudiantes de ingeniería y arquitectura de una universidad privada. Revista Muro de la Investigación [Internet]. 2020 [consultado el $25 \mathrm{Abr}$ 2020]; 5(1): 43-57. Disponible

en:

https://doi.org/10.17162/rmi.v5i1.1301

21.Reolid R, Flores M, López M, Alcantud P, Ayuso $C$, Escobar F. Frecuencia y características de uso de Internet por adolescentes españoles: Un estudio transversal. Arch. Argent. Pediatr [Internet]. 2016 [consultado 25 Abr 2020]; 114(1): 6-13. Disponible en: https://pesquisa.bvsalud.org/portal/resource/ pt/biblio-838159

22. Moral M, Suárez C. Factores de riesgo en el uso problemático de Internet y del teléfono móvil en adolescentes españoles. Revista Iberoamericana de Psicología y Salud [Internet]. 2016 [consultado 22 Abr 2020]; 7(2): 69-78. Disponible en: https://www.elsevier.es/esrevista-revista-iberoamericana-psicologiasalud-152-articulo-factores-riesgo-el-usoproblematico-S2171206916300011

23.Segura G, Neciosup J, Díaz G, Alcántara J, Díaz J. Uso de internet en actividades recreativas y sociales según perfil demográfico. Población adulta. Chimbote, 2017. UCV-SCIENTIA [Internet]. 2018 [consultado 26 Abr 2020]; 9(1): 64-64. Disponible en: http://revistas.ucv.edu.pe/index.php/UCVSCIENTIA/article/view/1503

24.Observatorio nacional de las telecomunicaciones y de la Sl. Perfil sociodemográfico de los internautas, análisis de datos INE 2018. Informe del equipo de estudios del ONTSI. Madrid: ONTSI; 2018. ISSN $2172-$ 9212. Disponible en: https://doi.org/10.30923/PerSoclnt-2018 
25. Hernández J, Ortiz M, Martínez J, Ramírez A, Miramontes A. Adicción a internet: el caso de adolescentes de cinco escuelas secundarias de México. Enseñanza e Investigación en Psicología [Internet]. 2019 [consultado $23 \mathrm{Abr}$ 2020]; 34-45. Disponible en: https://www.revistacneip.org/index.php/cneip /article/view/55

26.Domínguez P, Gonzáles H, Ramírez H. Efectos del uso excesivo de la internet en jóvenes universitarios. PsicoEducativa: Reflexiones $\mathrm{y}$ propuestas [Internet]. 2017 [consultado $22 \mathrm{Abr}$ 2020]; 3(6): 68-85. Disponible en: https://pdfs.semanticscholar.org/6106/11509 580ae35542ebf0caa5e53fa3d2e915e.pdf

27. Medrano P, Ramos M. Alteraciones cognitivas y emocionales en el insomnio crónico. Rev. Neurol [Internet]. 2016 [consultado $23 \mathrm{Abr}$ 2020]; 62(4): 170-178. Disponible en: https://www.neurologia.com/articulo/201533 4

28.Pons V. Internet la nueva era del delito: ciberdelito, ciberterrorismo, legislación y ciberseguridad. Urvio [Internet]. 2017 [consultado 25 Abr 2020]; (20): 80-93. Disponible en: http://200.41.82.22/handle/10469/12243

29.Rodríguez M, Rivedulla J. Percepción y experiencias sobre el ciberbullying en estudiantes universitarios. Revista d'innovación educativa [Internet]. 2018 [consultado $23 \mathrm{Abr}$ 2020]; (21): 10-22. Disponible en: https://www.redalyc.org/jatsRepo/3495/3495 57959002/349557959002.pdf 